\title{
ARTICLE
}

Received 3 Aug 2016 | Accepted 18 Nov 2016 | Published 22 Dec 2016

DOI: $10.1038 /$ ncomms 13988

OPEN

\section{Non-native three-dimensional block copolymer morphologies}

Atikur Rahman ${ }^{1}$, Pawel W. Majewski ${ }^{1}$, Gregory Doerk ${ }^{1}$, Charles T. Black ${ }^{1}$ \& Kevin G. Yager ${ }^{1}$

Self-assembly is a powerful paradigm, wherein molecules spontaneously form ordered phases exhibiting well-defined nanoscale periodicity and shapes. However, the inherent energyminimization aspect of self-assembly yields a very limited set of morphologies, such as lamellae or hexagonally packed cylinders. Here, we show how soft self-assembling materials-block copolymer thin films-can be manipulated to form a diverse library of previously unreported morphologies. In this iterative assembly process, each polymer layer acts as both a structural component of the final morphology and a template for directing the order of subsequent layers. Specifically, block copolymer films are immobilized on surfaces, and template successive layers through subtle surface topography. This strategy generates an enormous variety of three-dimensional morphologies that are absent in the native block copolymer phase diagram.

\footnotetext{
${ }^{1}$ Center for Functional Nanomaterials, Brookhaven National Laboratory, Upton, New York 11973, USA. Correspondence and requests for materials should be addressed to C.T.B. (email: ctblack@bnl.gov) or to K.G.Y. (email: kyager@bnl.gov).
} 
S elf-assembly has emerged as a prominent paradigm for the controlled formation of nanoscale-ordered phases $^{1-3}$. Block copolymer (BCP) thin films provide a straightforward means of rapidly and spontaneously generating uniform nanomaterials over macroscopic areas, where polymer architecture reliably encodes the nanoscale structural motif ${ }^{4,5}$. However, the inherent energy-minimization aspect of selfassembly emphasizes shapes that minimize surface area and maximize symmetry. Self-assembly thus yields a very limited set of morphologies, such as lamellae or hexagonally packed cylinders ${ }^{6,7}$. Here, we overcome this challenge, and demonstrate how soft self-assembling materials can be manipulated to form a diverse library of previously unreported morphologies. Non-equilibrium morphologies are accessed using a pathwaydependent assembly strategy. Our paradigm uses iterative assembly of BCP films, where each layer acts as both a structural component in the final morphology and a template that guides subsequent self-assembling layers. This is different from other layered-assembly approaches: one extreme is the simple stacking of independently ordered layers (layer-by-layer) ${ }^{8-14}$, which squanders the adaptive nature of self-assembly; on the other extreme is the direct replication of the first-layer pattern by subsequent layers (epitaxy) ${ }^{15-17}$, which cannot access non-native morphologies. Here, we present an intermediate 'responsive layering' approach that leverages the adaptive nature of soft materials, with each layer responding in a controlled fashion to those underneath. This strategy generates an enormous variety of three-dimensional (3D)morphologies that are not native to the bulk equilibrium BCP phase diagram.

\section{Results}

Responsive layering method. Our approach relies on sequential ordering and immobilization of two-dimensional (2D) BCP thin films in order to construct 3D morphologies in a layered fashion (Fig. 1). We spin-cast and anneal polystyrene-block-poly(methyl methacrylate) diblock copolymers (PS- $b$-PMMA) to yield ordered nanoscale phases ${ }^{5,18-20}$, and selectively infiltrate the PMMA block with alumina using a vapour-phase precursor (trimethylaluminium $)^{21,22}$. This selective infiltration synthesis (SIS) serves two roles: the infiltration 'fixes' the BCP film, rendering it insoluble and allowing additional polymer film applications atop; and preferential loading of the PMMA block with alumina swells this phase, generating subtle surface topography coincident with the morphology (1-4 nm; Supplementary Fig. 1). The fixed polymer-alumina composite film thus acts as a substrate for overlying assembly steps, leveraging the BCP's tendency to selfalign and register to underlying surface features. We spin-cast a neutralizing brush ${ }^{19,23-25}$, which prevents direct chemo-epitaxial replication of the underlying BCP pattern. The thin $(\sim 6 \mathrm{~nm})$ brush coats conformally and preserves the surface topography (Supplementary Figs 1-4). Thus, each BCP layer orders in response to the underlying topography, which is itself defined by self-assembly. This responsive layering can be repeated multiple times, building up a 3D morphology in a layered fashion. The entire structure can be converted into an inorganic alumina replica by ashing (exposure to $\mathrm{O}_{2}$ plasma, Fig. 1b,c). This methodology can rapidly generate intricate $3 \mathrm{D}$ nanostructures, including structures possessing cavities or voids in underlying layers (e.g., Fig. 1c).

New morphologies. This approach makes possible the creation of an enormous diversity of combined morphologies by marrying together BCP layers with different phases (cylinders, C; lamellae, $\mathrm{L}$; and inverse-cylinders, $\mathrm{O}$ ) and intrinsic repeat-periods ( $\mathrm{L}_{0}$, which is dictated by the copolymer molecular weight). Figure 2 shows scanning electron microscopy (SEM) images and schematics for all bilayer combinations assembling eight representative BCP materials (samples are designated by MXX, where $\mathrm{M}$ is the morphology and $\mathrm{XX}$ is the molecular weight). In addition to structures that resemble known BCP morphologies (e.g., hexagonally packed dots), a host of new morphologies appear. The most straightforward two-layer example is the

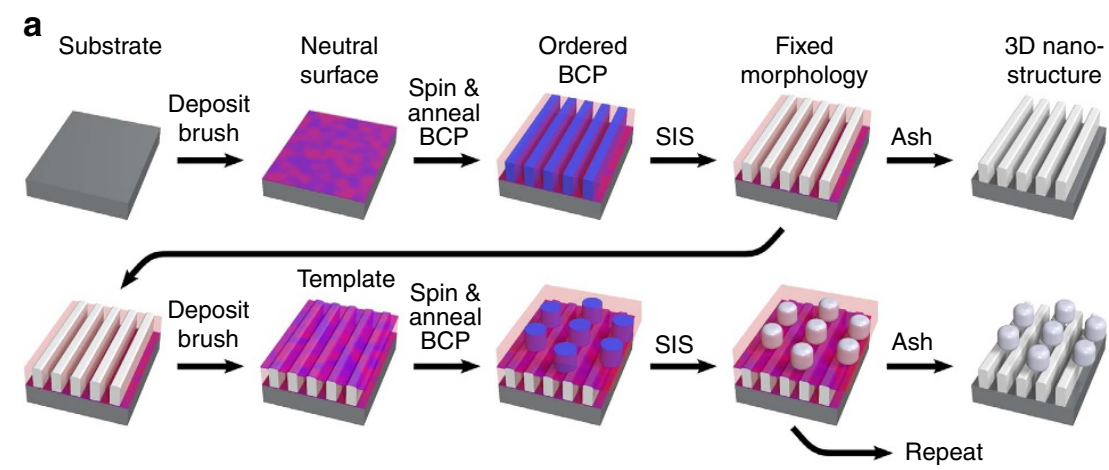

b

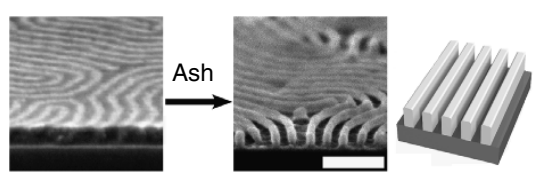

C

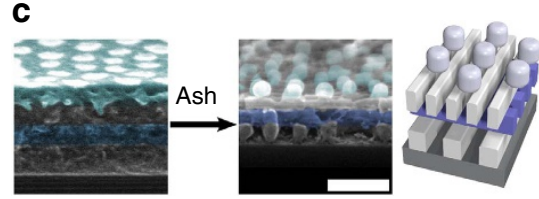

Figure 1 | Morphology assembly scheme. (a) The responsive assembly strategy begins by depositing a neutral brush on the substrate of interest. A block copolymer (BCP) film is then spin-coated on the substrate and annealed to yield a well-defined morphology. Selective infiltration synthesis (SIS) is used to load one of the BCP domains with alumina; this process 'fixes' the thin film, making it robust and insoluble, and generates height variation between the domains. The fixed BCP film can be used as the substrate in a subsequent round of ordering. The height variation (which remains after depositing the neutral brush) templates the subsequent BCP layer, causing it to align and register in a well-defined way. Single-layer, bi-layer or multilayer inorganic replicas can be formed by ashing the film (exposure to $\mathrm{O}_{2}$ plasma), as shown in the right-most column. (b) Cross-sectional scanning electron microscopy (SEM) of an exemplar single-layer infiltrated nanostructure before and after ashing (lamellar-forming BCP, L36). (c) Cross-sectional SEM of an exemplar four-layer nanostructure. False-colour applied to highlight layers (from bottom to top): L104, L74, L36, C132. Scale bars are $100 \mathrm{~nm}$. 
a

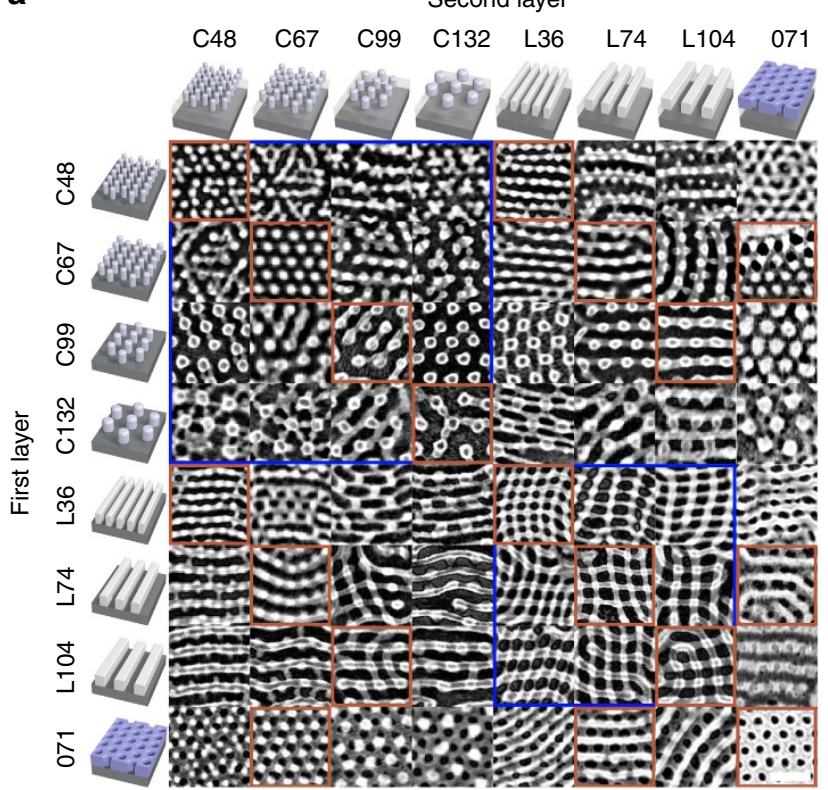

b

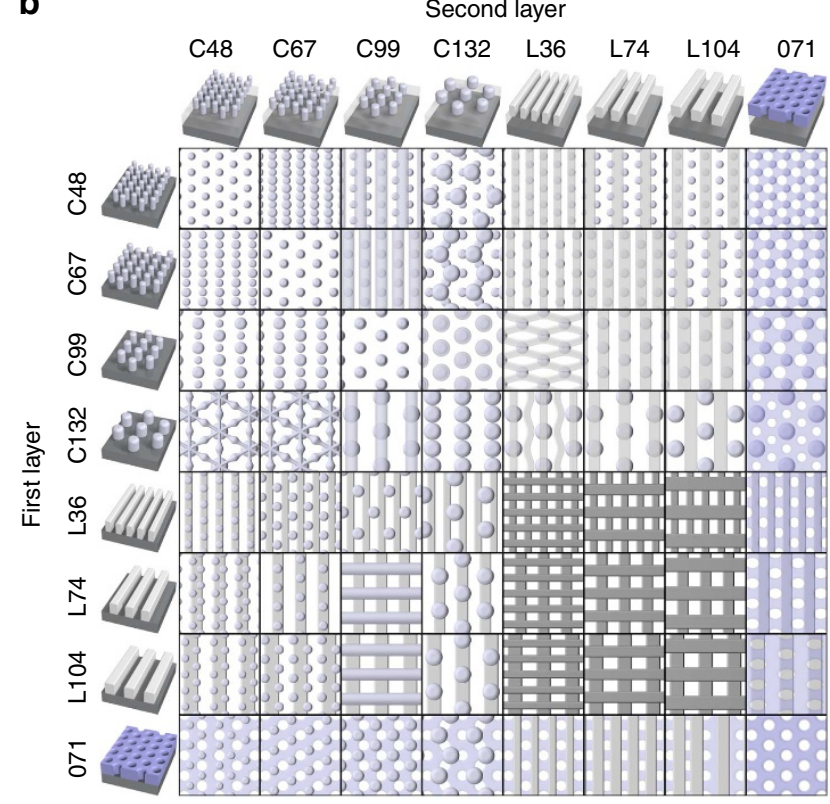

Figure 2 | Diversity of morphologies. (a) Scanning electron microscopy (SEM) images of two-layer nanostructures formed by the iterative assembly of block copolymer films. Scale bar is $100 \mathrm{~nm}$, and applies to all images. The ordering of the second layer is templated by the morphology of the first layer, giving rise to combined morphologies not native to the bulk equilibrium BCP phase diagram. The blue boxes highlight areas where the same class of morphology is used in both layers; the red boxes highlight conditions where the lattice spacings of the two layers are roughly equal. (b) Schematics of the idealized three-dimensional assemblies formed, highlighting the enormous diversity of morphologies that can be accessed.

successive applications of the same cylinder-forming material, where the second layer of vertical cylinders self-registers epitaxially to the first (e.g., C67-C67). The combined structure thus exhibits the same symmetry as the component materials (p6mm symmetry, with respect to the projected $2 \mathrm{D}$ order). The successive application of the same morphology can also yield structures distinct from the components. For lamellae, the second-layer material preferentially anti-aligns with respect to the first, giving rise to square (e.g., L74-L74, p4mm symmetry) or rectangular (e.g., L36-L104, p2mm symmetry) patterns. Combinations of disparate morphologies can give rise to new surprising structures. For instance, ordering of lamellar L74 on top of the hexagonal C48 material yields stripes interposed through every second pair of cylinder rows, with the cylinders forming a 'zig-zag' motif (p2mg symmetry). The library of bilayer morphologies obtained by combining cylinder, lamellar and inverse cylinders shows a significantly greater degree of complexity compared with the three simple component morphologies (Fig. 2b and Supplementary Fig. 5). The combined morphologies include examples of 7 different wallpaper symmetry groups (out of the 17 possible), and display significant structural diversity within a particular symmetry group (Supplementary Fig. 6). Importantly, the combined morphologies frequently exhibit symmetry and structural motifs not present in either of the constituent layers (Supplementary Fig. 7), emphasizing that the obtained structures are self-assembled and emergent.

A key feature in the observed assembly is the well-defined registry between successive layers, even when combining disparate morphologies. For instance, when C48 assembles on L36, the cylinder cores are positioned exactly on top of the underlying PMMA/alumina stripes (Fig. 3a). Assembly of C48 on top of larger repeat-period lamellae results in the second-layer cylinders aligning on the edge between the PMMA/alumina and PS regions, resulting in zig-zag patterns (Fig. 3b). It is clear that the second layer is not ordering independently from the first. For instance, when a large repeat-period cylinder phase (C132) orders on a small repeat-period lamellar pattern (L36), the second-layer cylinders register in-between the first-layer stripes, occupying every other interstice, and maintaining local hexagonal cylinder packing (Fig. 3d). This ordering is conserved over macroscopic sample dimensions, as confirmed by Fourier transforms of wide-area SEM images (Supplementary Figs 8 and 9) and grazing-incidence X-ray scattering (Fig. 3e and Supplementary Figs 10-12). The registration of layers requires distortion of the second-layer repeat-period when the two materials are not commensurate. For instance, L74 stretches its period to register within every second row of a C48 hexagonal lattice, adopts its bulk equilibrium period to align with the rows of C67 material and stretches to remain 1:1 overlapped with the larger spacing of C99 and C132 (confirmed using Fourier analysis; Supplementary Fig. 9). Overall, the registered self-assembly of two-layer nanostructures gives rise to a host of non-native morphologies (see Supplementary Figs 13-20 for examples). For instance, the organization of L36 on C99 (or C132) gives rise to undulating stripes that 'cross-connect' between cylinders. Fourier analysis confirms the suppression of the pure L36 ordering, and the emergence of a distinct combined unit cell (Supplementary Figs 13-15).

Mechanism of responsive layering. We understand this unique ordering behaviour by noticing that the second BCP layer tends to maximize the overlap of its interblock interfaces to the interblock interfaces in the underlying layer. Owing to the SIS-induced swelling of the PMMA domain, the interblock interface is where maximum height variation arises. Thus, the second-layer BCP orders so as to overlap its interblock region with the underlying height variation. In the L36-C48 assembly, the second-layer cylinders align with the first-layer stripes to maximize the overlap of the cylinder edges with the stripe edges. For L104-C48, the C48 domains sit just off the edges, with the stripes running in between, as this maximizes the overlap of the C48 cylinder perimeters with the underlying height variation. This registration 
a

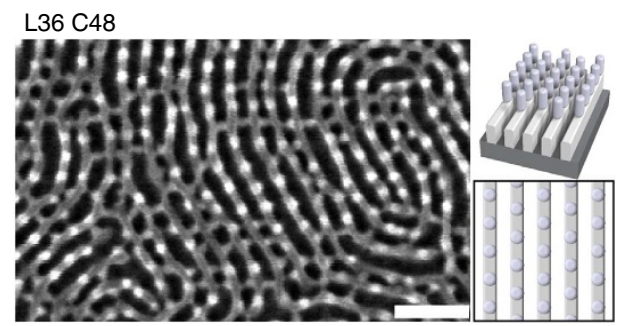

b

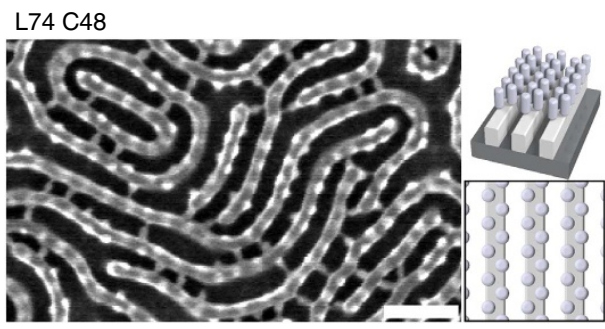

C

d

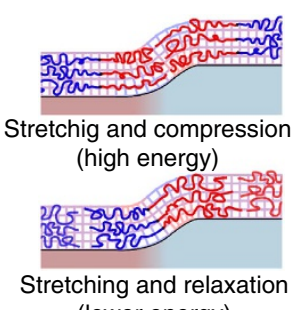
(lower energy)

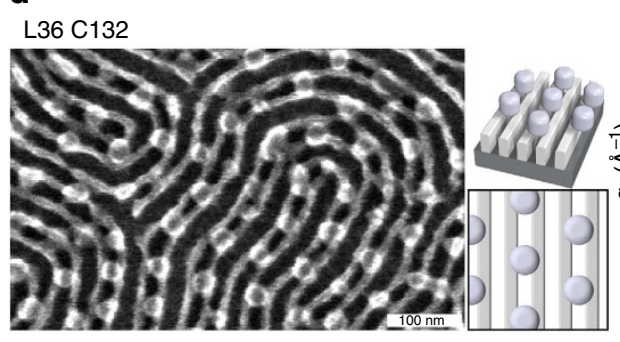

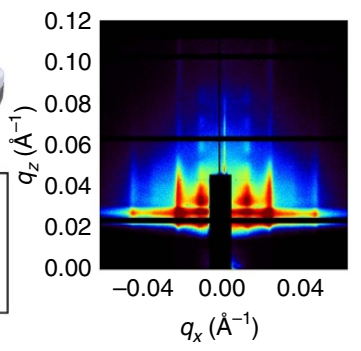

$q_{x}\left(\AA^{-1}\right)$

e

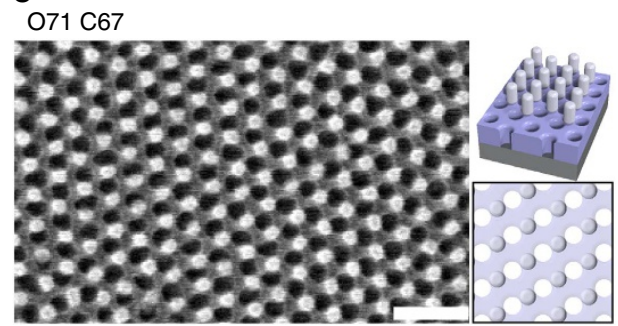

f

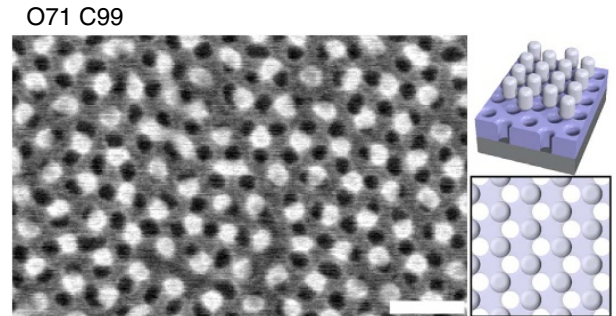

Figure 3 | Templated ordering. (a) Commensurate assembly of a cylinder material atop a lamellar involves the cylinders organizing along the underlying stripes. (b) When an incommensurate cylinder phase orders on top of a larger repeat-period lamellar pattern, the cylinder row-spacing distorts so as to align the cylinders with the edges of the underlying stipes. (c) This registry phenomena can be understood in terms of chain distortion. If the central region of a BCP domain is positioned on top of a height variation, the BCP chains must stretch and compress. If instead the interblock interface is positioned over the height variation, some BCP chains stretch, while other stretched chains are unstretched (relax), leading to a lower overall energy. (d) This registration can be seen in C132 ordering atop L36, where the edges of cylinders align with underlying stripes. Corresponding X-ray scattering demonstrates the templated order over wide areas. (e) Nearly-commensurate cylinders ordering atop a honeycomb pattern results in two offset hexagonal lattices, again maximizing overlap of the interblock interface with the height variation. (f) Slightly incommensurate ordering exhibits similar registry, but with higher defect density. Scale bars are $100 \mathrm{~nm}$.

phenomenon can be explained in terms of BCP chain stretching (Supplementary Discussion and Supplementary Figs 21-24). When a $\mathrm{BCP}$ film orders on a weak $\left(<\mathrm{L}_{0}\right)$ topographic pattern, it will bend to conformally coat the substrate, generating an internal stress field. Bending involves a combination of polymer chain stretching and compression (Supplementary Fig. 22). The chains within an ordered BCP mesophase are stretched, with the segments near the interblock interface more stretched than those nearer the chain ends ${ }^{26-29}$. Bending a BCP in the centre of one of the domains involves stretching and compressing relatively unperturbed chains; both of these distortions incur an energy penalty. In contrast, bending a $\mathrm{BCP}$ at the interblock domain boundary involves distorting chains that are already highly stretched. Further stretching involves an additional energy penalty; however, compression of stretched chains relaxes them toward their unperturbed conformation (Supplementary Fig. 21). Thus, the overall energy penalty for distorting the $\mathrm{BCP}$ domain boundary is less than distorting other regions of the morphology. This results in the $\mathrm{BCP}$ organizing so as to maximize the overlap between substrate height modulations and the domain boundary region of the morphology, since this overall lowers the chain distortion energy penalty (Fig. 3c).
This heuristic explains the rich behaviour observed in combining a wide variety of morphologies and size scales. For example, lamellar domains orient perpendicularly to underlying lamellar morphologies (Fig. 2) ${ }^{30-32}$. This 'crossed' alignment completely eliminates stretching/compression of the polymer chains along the long axis of the coil (i.e., normal to the lamellar interblock interface). In this perpendicular orientation, the lamellar chains can accommodate the underlying height variation by simple rearrangement of chain packing orthogonal to the coil long axis. Such a simple accommodation is not possible for cylinder morphologies, since polymer chains are arranged across all inplane orientations. For cylinder second layers, the morphology orients and registers so as to maximize the overlap of the interblock interface with underlying height variation (to minimize chain distortion along the coil long axis). Cylinder-forming material (C67 or C99) ordering atop a slightly incommensurate honeycomb pattern (O71) shifts so as to overlap cylinder perimeters with the underlying height variation, resulting in two offset hexagonal lattices (Fig. 3e,f). Greater mismatch in period can introduce a relative rotation of the two lattices (Supplementary Fig. 20).

BCP assembly is known to be responsive to confinement ${ }^{33-35}$, guiding trenches ${ }^{36-38}$ (i.e., grapho-epitaxy ${ }^{39,40}$ ), topographic 
disruptions $^{41-44}$, and substrate roughness ${ }^{45,46}$, corrugations ${ }^{30-32}$ or faceting ${ }^{47-49}$. BCPs typically respond according to commensurability, reorienting and distorting to preserve their bulk equilibrium morphology. Our work suggests a subtler variant, where the $\mathrm{BCP}$ not only distorts to be commensurate with the underlying pattern but also registers to weak topography in order to minimize chain perturbation. The full diversity of structures we observe (Fig. 2) can be explained using a cascade of chain distortion effects: the repeat-period may distort to become commensurate with the under-layer; the morphology adopts an orientation and registry to minimize chain distortion by placing interblock interfaces atop height variations; finally, in cases of degenerate configurations, the material with a lower bending energy penalty will sit on top of height variations. These effects are evidently strong enough to distort the BCP far from its bulk equilibrium morphology (c.f. undulating lines of C99-L36 or bridging-bulges of C132-C67). It is also possible that for some morphologies, the final development steps (infiltration and ashing) play a role in accentuating, or selecting, the observed features.

Film thickness influences the ordering phenomena observed herein. It is well-known that BCP films are strongly responsive to film thickness ${ }^{1,4,5,50-52}$, with the morphology or orientation being influenced by interfaces and commensurability considerations (Supplementary Fig. 25). For the results presented here, the film thickness was monolayer or sub-monolayer (with respect to the BCP repeat-distance), which drives ordering towards a vertical orientation of the morphology (cylinder long axis perpendicular to substrate; lamellar sheets perpendicular to substrate). This ordering propensity can be confirmed by noting that the first layer in the bilayer morphologies (Fig. 2) all exhibit vertical orientations. The same spin-casting conditions were used for the second layer of the bilayer structures. In general, these secondlayer materials also adopt a vertical orientation. However, a number of counterexamples can be seen (e.g., C67-C99), which indicates that the responsive layering phenomenon observed here is sufficiently strong to overcome thin film confinement effects. The orientation and order (defect density) of the second-layer material can be tuned by adjusting the film thickness. Thus, film thickness is an additional parameter that can be used to control ordering of multilayer BCP nano-structures.

The presented energy model ignores many pertinent aspects of $\mathrm{BCP}$ response, including interplay with film thickness, and the potential formation of different morphologies to minimize chain distortion. These possibilities could be captured by more sophisticated modelling. Field-theoretic methods have emerged as a powerful tool for understanding the assembly of $\mathrm{BCP}$ phases $^{6}$, and it is likely that their application to the materials described herein could yield new insights. For instance, selfconsistent field theory was used to demonstrate that relatively weak surface corrugations could strongly influence BCP assembly, selecting among different possible morphologies ${ }^{49}$. These simulations help to confirm the heuristic presented here. That is, subtle substrate topography can control the registration of a BCP film. Simulations of BCP ordering under more extreme topographic confinement predict the appearance of 3D motifs very distinct from bulk ordering ${ }^{53-56}$, suggesting that elaborated extensions of the methods presented herein will yield an even greater diversity of nanostructures.

An important consideration in assessing self-assembling nanomaterials is their defectivity. Conventional BCP materials (single-component thin film) exhibit substantial defect densities. The topological defects in BCP phases (disclinations, dislocations, grain boundaries) are high energy ${ }^{57,58}$; at equilibrium the expected areal defect density would be very low. The much larger defect densities observed experimentally are thus metastable, arising from the ordering history, rather than equilibrium fluctuations ${ }^{59}$. While the local ordering (morphology) is determined thermodynamically, the defectivity is kinetic. We observe similar phenomena in our multilayer assembled morphologies. The local ordering motifs can be ascribed to equilibrium effects, where the layer relaxes into the (local) energy minimum. On the other hand, the defect density is kinetically limited (e.g., can be reduced with annealing time). In our responsive layering scheme, defects can arise in three different ways. Firstly, the BCP materials exhibit the same inherent defectivity as conventional single-layer BCP films; thus each layer will exhibit a certain density of kinetically limited defects. Additionally, the second layer will have a higher defect density, since it inherits defects from the first layer; that is, any defect in the first layer will disrupt the preferred local motif, introducing a defect in the second layer. Finally, incompatibility between the morphologies in subsequent layers may frustrate ordering, generating new defects; for instance, incommensurability will cause an overlayer to distort in order to register to underlying height variations, with the associated internal stress increasing the probability of defects. Indeed, we observe that commensurate combinations (e.g., O71-C67) exhibit long-range order, while incommensurate combinations have increased defect densities.

The acceptable level of defectivity of course depends on the target application. For some applications, long-range order is crucial, whereas for others, well-defined local morphology is sufficient. For example, tuning bulk optical and electrical properties, or generating membranes for nano-filtration or catalysis, requires optimization of the local nanoscale structure, but is insensitive to long-range order. On the other hand, applications in nano-electronics or data storage require exceptionally low defect densities over large areas. The materials presented herein should immediately be useful for a wide variety of nano-materials applications. Where lower defectivity is necessary, the presented assembly strategy could easily be combined with established directed self-assembly methods, which can rapidly generate aligned BCP phases with considerably lower defectivity 20 . An exciting avenue for future investigation is to study the interplay between directed self-assembly methods and the responsive layering described herein.

The presented mechanistic explanation provides means of predictably generating desired three-dimensional nanostructures. Combining commensurate $\mathrm{BCP}$ materials (1:1, 1:2, etc.) will minimize defectivity, and will give rise to nanostructures where the structures of the individual phases are preserved and wellregistered to one another, thus forming a distinct overall symmetry. Combining slightly incommensurate layers will give rise to new, distorted morphologies (e.g., undulating lines), while highly incommensurate combinations may yield poorly ordered structures. Generally speaking, a size-mismatch between the nanostructures in the two layers will give rise to 'out-of-phase' combined structures, where the second-layer structure (e.g., cylinder cores) sit in between the first-layer structures. Conversely, layering of similar-sized nanostructures gives rise to 'in-phase' combinations, where the structures in the layers align on top of one another. We note that the mechanism is not dependent on system-specific properties, and should thus manifest for any BCP materials. Indeed, we confirmed similar behaviour in the assembly of polystyrene-block-poly(2-vinyl pyridine) BCPs (Supplementary Fig. 26).

\section{Discussion}

The presented method allows the rapid formation of an enormous diversity of 3D morphologies. There has been substantial progress in expanding the range of self-assembled shapes through 
L36 L74 L104
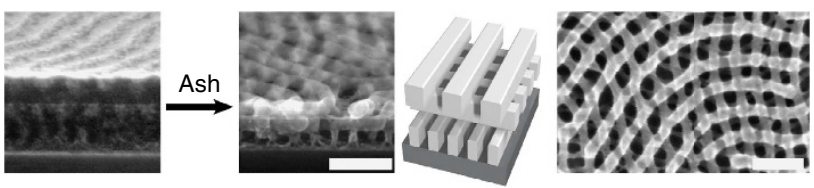

O71 L74 C67
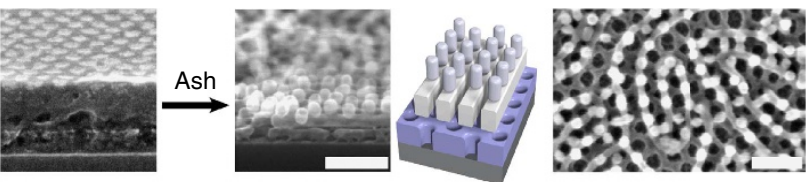

O71 L36 C132
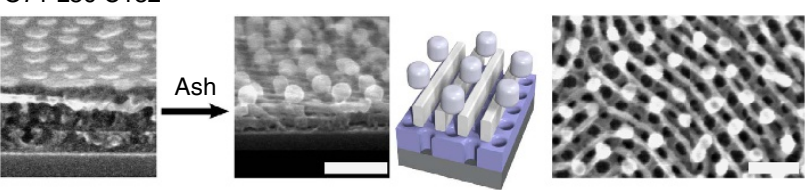

O71 L36 L74 L104
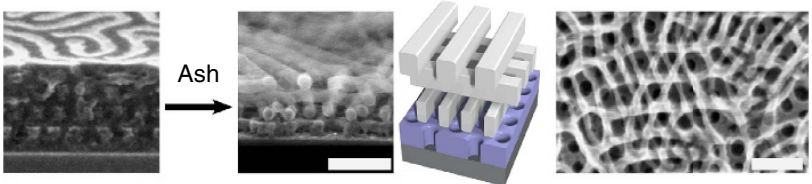

L104 L74 L36 C132
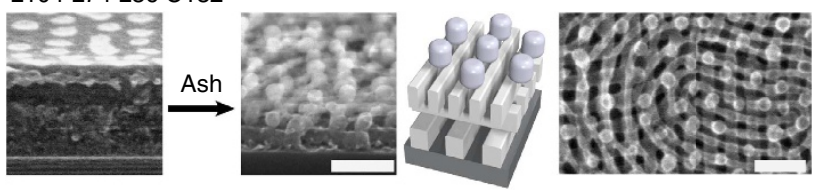

Figure 4 | Multilayered 3D nanostructures. Examples of 3D nanostructures formed by layered block copolymer assembly. The order of layers (from bottom to top) is given for each row. The post-ash materials are clearly nanoporous, with underlying channels and voids. Scale bars are $100 \mathrm{~nm}$.

materials synthesis (e.g., triblocks ${ }^{60-62}$ ) and preparation (e.g., blending $\left.{ }^{61,63,64}\right)$. Directed assembly using lithographic templates can also promote the formation of distinct patterns ${ }^{42,65-67}$. Our iterative self-assembly method allows morphologies to be explored rapidly and combinatorially, by simple layering of existing materials. Conventional layer-by-layer or epitaxial approaches allow straightforward prediction and control of structure formation, but sacrifice many of the inherent advantages of selfassembly. Conversely, our method exploits the adaptive nature of self-assembly to generate surprising non-equilibrium morphologies. Our responsive layering paradigm can construct a wide variety of complex morphologies over wide areas, including threedimensional nanostructures containing underlying channels or pores (Fig. 4). At each layer, the ordering occurs spontaneously, resulting in a controlled, templated structure; thus, one can select whether underlying features are exposed or covered. We note that it would be challenging to construct similar structures using conventional self-assembly, or even advanced lithographic techniques. This level of control opens the door to a wide variety designed materials, and thus broadens the range of applications that can be targeted.

\section{Methods}

Polymer materials. Polystyrene-block-poly(methyl methacrylate) (PS-b-PMMA) block copolymers of various molecular weights (ranging from 48 to $132 \mathrm{~kg} \mathrm{~mol}^{-1}$ ) were obtained from Polymer Source Inc; a subset of materials were characterized by nuclear magnetic resonance spectroscopy, and gel permeation chromatography to confirm composition and purity. Throughout this work, we use a sample code MXX, where M denotes the morphology (L, lamellar; C, cylinder phase with PMMA as minority; $\mathrm{O}$, inverse cylinder phase with PMMA as matrix) and $\mathrm{XX}$ denotes the total molecular weight (in $\mathrm{kg} \mathrm{mol}^{-1}$ ). For example, C48 refers to a cylinder-forming PS- $b$-PMMA (PS matrix, PMMA cylinder cores) of total molecular weight $48 \mathrm{~kg} \mathrm{~mol}^{-1}\left(31.6 \mathrm{~kg} \mathrm{~mol}^{-1} \mathrm{PS}, 17.5 \mathrm{~kg} \mathrm{~mol}^{-1}\right.$ PMMA, polydispersity $M_{\mathrm{w}} / M_{\mathrm{n}}=1.06$ ), which exhibits a repeat-period of $L_{0}=26 \mathrm{~nm}$.

Substrates. In order to control the domain orientation relative to the substrate and air interfaces, silicon substrate surfaces were neutralized by applying a PS- $r$-PMMA-OH random copolymer (provided by the Dow Chemical Company), which acts as a brush that is chemically 'neutral' (similar interaction energy with PS and PMMA). The brush composition was $61 \mathrm{~mol} \%$ styrene, determined by ${ }^{13} \mathrm{C}$ NMR, with total molecular weight $M_{\mathrm{n}}=9.2 \mathrm{~kg} \mathrm{~mol}^{-1}$ and polydispersity $M_{\mathrm{w}} / M_{\mathrm{n}}=1.35$ (determined by gel permeation chromatography relative to PS standards). The neutral brush was applied by spin coating at 750 r.p.m., followed by annealing at $220^{\circ} \mathrm{C}$ for $5 \mathrm{~min}$ to promote adhesion and finally rinsed in toluene to remove excess material. This protocol results in a deposited brush layer of $\sim 6 \mathrm{~nm}$ (based on spectroscopic ellipsometry).

Polymer films. BCP thin films were prepared by spin casting $1 \%(\mathrm{wt} / \mathrm{wt})$ toluene solution of PS- $b$-PMMA onto polished silicon (100) substrates with neutral brush coating. These coating conditions yield a polymer film thickness of $20-30 \mathrm{~nm}$ (depending on material), which is in the monolayer or sub-monolayer regime. A consistent spin-casting protocol was used for all the layers described in the main text. This enables unambiguous cross-comparison of results. We note, however, that the ordering of a particular multilayered morphology could be optimized by adjusting the film thicknesses of the various layers. BCP samples were annealed at $220^{\circ} \mathrm{C}$ for $5 \mathrm{~min}$ on a hot plate to yield ordered morphologies. Cylindrical phase PS- $b$-PMMA ( 70:30 PS:PMMA weight ratio) self-assemble into locally ordered hexagonal arrangements of PMMA cylinders within a PS matrix (denoted C). Owing to the neutral substrate, the cylinder long axis is oriented perpendicular to the substrate plane. Opposite tone or inverse-cylinder PS-b-PMMA with 20:51 PS:PMMA weight ratio (O71 material) generates hexagonal patterns of PS cylinders in PMMA matrix. Finally, 50:50 weight ratio PS- $b$-PMMA generates lamellar (L) morphologies, which orient with the lamellar sheets perpendicular to the substrate plane (owing to the neutral brush) and thus appears as line/space ('fingerprint') patterns. As a control experiment, a subset of bilayer morphologies were ordered with different annealing conditions (varying temperature and/or annealing time). In such cases, the final local ordering motifs (morphology of the multilayer nanostructure) were found to be identical, although the defect density was variable. This suggests that the structure formation is driven by equilibrium effects (energy minimization), while defectivity is a result of kinetic trapping and slow coarsening kinetics (precisely consistent with well-known single-layer BCP thin film ordering results).

Material conversion. BCP films were immobilized by exposure to vapour phase precursors. Polymer films can alternatively be immobilized via crosslinking $8,9,16,17,68,69$; however, a key feature of our method is to exploit infiltration to generate surface height variation coincident with the morphology. The PMMA regions of the self-assembled BCP patterns were transformed into metal oxide nanostructures using SIS; that is, by sequential exposure to a suitable vapour-phase organometallic precursor and water vapour ${ }^{21,22}$. In our case, samples were exposed to four sequential cycles of trimethylaluminium followed by water vapour, using Cambridge Nanotech atomic layer deposition tool ( 2 min exposures to $\sim 10$ Torr of both trimethylaluminium and water at $85^{\circ} \mathrm{C}$ ) to convert the PMMA regions into aluminium oxide. The infiltration of PMMA regions causes swelling of these domains, leading to the formation of a $1-4 \mathrm{~nm}$ topography coincident with the morphology (as confirmed by scanning probe microscopy).

Multilayers. To fabricate multilayered structures, the infiltrated samples were used as a substrate for the above-described process. Crucially, the infiltrated BCP is robust and insoluble, allowing spin-coating from toluene to be used to deposit additional layers. Thus, the infiltrated BCP material has a neutral brush deposited using spin-coating and baking, followed by deposition of an additional BCP layer by spin-coating and annealing. This multilayered system can be infiltrated and used again as the substrate for further layering. The neutral brush deposited onto an infiltrated BCP layer is uniform, despite the chemical inhomogeneity of the BCP top surface. This was confirmed based on AFM height measurements (refer to Supplementary Fig. 1), where the height variation of a brush-coated material $(2-4 \mathrm{~nm})$ is less than the expected thickness of the brush layer $(6-7 \mathrm{~nm})$. This likely occurs because the brush material is able to adsorb to both PS and PMMA/ $\mathrm{AlO}_{x}$ regions, and may be facilitated by a partial and ultra-thin $\mathrm{AlO}_{x}$ layer that incidentally coats even the PS regions (owing to a generic ALD process). Such an ultra-thin layer would decrease chemical inhomogeneity, while not impeding eventual removal of the PS domains via ashing. 
Ashing. After multilayer structure fabrication, the organic BCP templates were removed using oxygen plasma etching $(20 \mathrm{~W}, 100 \mathrm{mTorr}$, for $3 \mathrm{~min}$ at room temperature). This process removes the organic material, leaving behind an inorganic replica of the ordered PMMA domains. Multilayered materials can be ashed with the same protocol; SEM imaging confirms that organic material is removed from all layers.

Imaging. Samples were imaged using SEM in a Hitachi S-4800 instrument Surface topography was quantified using scanning probe microscopy in intermittent-contact ('tapping') mode, at $1 \mathrm{~Hz}$ scanning rate, on an Asylum MFP-3D instrument. Scanning probe microscopy tips (MikroMash HQ:NSC14/Al BS) were $\mathrm{Si}, 8 \mathrm{~nm}$ tip radius, with resonant frequency $160 \mathrm{kHz}$ (spring constant $5.0 \mathrm{~N} \mathrm{~m}^{-1}$ ).

GISAXS. Grazing-incidence small-angle X-ray scattering ${ }^{70}$ measurements were performed at the 11-ID CHX (Coherent Hard X-ray) beamline of the National Synchrotron Light Source II, at Brookhaven National Laboratory. Samples were measured under vacuum using an X-ray beam of $8.8984 \mathrm{keV}(\lambda=0.1393 \mathrm{~nm})$. Grazing-incidence small-angle X-ray scattering data presented were collected across a range of incidence angles $\left(0.13-0.21^{\circ}\right)$. Silver behenate powder was used as a standard for data conversion to $q$-space. Beam size was approximately $20 \mu \mathrm{m} \times 20 \mu \mathrm{m}$, thereby probing a film area of $(20 \mu \mathrm{m}) \times(6.7 \mathrm{~mm}) \approx 0.1 \mathrm{~mm}^{2}$ in grazing-incidence geometry.

Data availability. The data that support the findings of this study are available from the corresponding author upon reasonable request.

\section{References}

1. Segalman, R. A. Patterning with block copolymer thin films. Mater. Sci. Eng. $R$ 48, 191-226 (2005)

2. Bang, J., Jeong, U., Ryu, D. Y., Russell, T. P. \& Hawker, C. J. Block copolymer nanolithography: translation of molecular level control to nanoscale patterns. Adv. Mater. 21, 4769-4792 (2009).

3. Ross, C. A., Berggren, K. K., Cheng, J. Y., Jung, Y. S. \& Chang, J.-B. Threedimensional nanofabrication by block copolymer self-assembly. Adv. Mater. 26, 4386-4396 (2014).

4. Fasolka, M. J. \& Mayes, A. M. Block copolymer thin films: physics and applications. Annu. Rev. Mater. Res. 31, 323-355 (2001).

5. Albert, J. N. L. \& Epps, T. H. Self-assembly of block copolymer thin films. Mater. Today 13, 24-33 (2010).

6. Bates, F. S. \& Fredrickson, G. H. Block copolymer thermodynamics: theory and experiment. Annu. Rev. Phys. Chem 41, 525-557 (1990).

7. Castelletto, V. \& Hamley, I. W. Morphologies of block copolymer melts. Curr. Opin. Solid State Mater. Sci. 8, 426-438 (2004).

8. Ruiz, R., Sandstrom, R. L. \& Black, C. T. Induced orientational order in symmetric diblock copolymer thin films. Adv. Mater. 19, 587-591 (2007).

9. Rose, F., Bosworth, J. K., Dobisz, E. A. \& Ruiz, R. Three-dimensional mesoporous structures fabricated by independent stacking of self-assembled films on suspended membranes. Nanotechnology 22, 035603 (2011).

10. Jeong, J. W. et al. Nanotransfer printing with sub-10 nm resolution realized using directed self-assembly. Adv. Mater. 24, 3526-3531 (2012).

11. Kim, J. Y. et al. Flexible and transferrable self-assembled nanopatterning on chemically modified graphene. Adv. Mater. 25, 1331-1335 (2013).

12. Kim, S. Y. et al. Large-area nanosquare arrays from shear-aligned block copolymer thin films. Nano Lett. 14, 5698-5705 (2014).

13. Jeong, J. W. et al. High-resolution nanotransfer printing applicable to diverse surfaces via interface-targeted adhesion switching. Nat. Commun. 5 (2014).

14. Majewski, P. W., Rahman, A., Black, C. T. \& Yager, K. G. Arbitrary lattice symmetries via block copolymer nanomeshes. Nat. Commun. 6, 7448 (2015).

15. Park, S.-M. et al. Combinatorial generation and teplication-directed assembly of complex and varied geometries with thin films of diblock copolymers. Langmuir 23, 9037-9045 (2007).

16. Kim, E. et al. Size control and registration of nano-structured thin films by cross-linkable units. Soft Matter 4, 475-479 (2008).

17. He, C. \& Stoykovich, M. P. Profile control in block copolymer nanostructures using bilayer thin films for enhanced pattern transfer processes. Adv. Funct. Mater. 24, 7078-7084 (2014).

18. Hu, H., Gopinadhan, M. \& Osuji, C. O. Directed self-assembly of block copolymers: a tutorial review of strategies for enabling nanotechnology with soft matter. Soft Matter 10, 3867-3889 (2014).

19. Stein, G. E., Mahadevapuram, N. \& Mitra, I. Controlling interfacial interactions for directed self assembly of block copolymers. J. Polym. Sci. B 53, 96-102 (2014).

20. Majewski, P. W. \& Yager, K. G. Rapid ordering of block copolymer thin films. J. Phys. Condens. Matter 28, 403002 (2016).

21. Peng, Q., Tseng, Y.-C., Darling, S. B. \& Elam, J. W. Nanoscopic patterned materials with tunable dimensions via atomic layer deposition on block copolymers. Adv. Mater. 22, 5129-5133 (2010).
22. Ramanathan, M., Tseng, Y.-C., Ariga, K. \& Darling, S. B. Emerging trends in metal-containing block copolymers: synthesis, self-assembly, and nanomanufacturing applications. J. Mater. Chem. C 1, 2080-2091 (2013)

23. Mansky, P., Liu, Y., Huang, E., Russell, T. P. \& Hawker, C. Controlling polymer-surface interactions with random copolymer brushes. Science $\mathbf{2 7 5}$, 1458-1460 (1997).

24. Huang, E., Rockford, L., Russell, T. P. \& Hawker, C. J. Nanodomain control in copolymer thin films. Nature 395, 757 (1998).

25. Han, E. et al. Perpendicular orientation of domains in cylinder-forming block copolymer thick films by controlled interfacial interactions. Macromolecules $\mathbf{4 2}$, 4896-4901 (2009).

26. Almdal, K., Rosedale, J. H., Bates, F. S., Wignall, G. D. \& Fredrickson, G. H. Gaussian- to stretched-coil transition in block copolymer melts. Phys. Rev. Lett. 65, 1112-1115 (1990).

27. Binder, K. \& Fried, H. Asymmetric block copolymer melts near the microphase separation transition: a Monte Carlo simulation. Macromolecules 26, 6878-6883 (1993).

28. Lee, N.-K., Johner, A. \& Vilgis, T. A. Single chain stretching of block copolymers under different solvent conditions. Macromolecules 35, 6043-6054 (2002).

29. Forrey, C., Yager, K. G. \& Broadaway, S. P. Molecular dynamics study of the role of the free surface on block copolymer thin film morphology and alignment. ACS Nano 5, 2895-2907 (2011).

30. Ho-Cheol, K., Charles, T. R. \& Linnea, S. Fabrication of 20nm half-pitch gratings by corrugation-directed self-assembly. Nanotechnology 19, 235301 (2008).

31. Park, S. M., Berry, B. C., Dobisz, E. \& Kim, H. C. Observation of surface corrugation-induced alignment of lamellar microdomains in PS-b-PMMA thin films. Soft Matter 5, 957-961 (2009).

32. Tavakkoli, K. G. et al. Multilayer block copolymer meshes by orthogonal selfassembly. Nat. Commun. 7 (2016).

33. Wu, Y. et al. Composite mesostructures by nano-confinement. Nat. Mater. 3, 816-822 (2004).

34. Jung, Y. S., Jung, W. \& Ross, C. A. Nanofabricated concentric ring structures by templated self-assembly of a diblock copolymer. Nano Lett. 8, 2975-2981 (2008).

35. Yabu, H., Higuchi, T. \& Jinnai, H. Frustrated phases: polymeric self-assemblies in a 3D confinement. Soft Matter 10, 2919-2931 (2014).

36. Segalman, R. A., Hexemer, A. \& Kramer, E. J. Effects of lateral confinement on order in spherical domain block copolymer thin films. Macromolecules 36, 6831-6839 (2003).

37. Sundrani, D., Darling, S. B. \& Sibener, S. J. Guiding polymers to perfection: macroscopic alignment of nanoscale domains. Nano Lett. 4, 273-276 (2003)

38. Ruiz, R., Ruiz, N., Zhang, Y., Sandstrom, R. L. \& Black, C. T. Local defectivity control of 2D self-assembled block copolymer patterns. Adv. Mater. 19, 2157-2162 (2007).

39. Cheng, J. Y., Ross, C. A., Smith, H. I. \& Thomas, E. L. Templated self-assembly of block copolymers: top-down helps bottom-up. Adv. Mater. 18, 2505-2521 (2006).

40. Koo, K., Ahn, H., Kim, S.-W., Ryu, D. Y. \& Russell, T. P. Directed self-assembly of block copolymers in the extreme: guiding microdomains from the small to the large. Soft Matter 9, 9059-9071 (2013).

41. Zhang, X. et al. Target patterns induced by fixed nanoparticles in block copolymer films. ACS Nano 3, 2115-2120 (2009).

42. Tavakkoli, K. G. et al. Templating three-dimensional self-assembled structures in bilayer block copolymer films. Science 336, 1294-1298 (2012).

43. Shin, D. O. et al. Multicomponent nanopatterns by directed block copolymer self-assembly. ACS Nano 7, 8899-8907 (2013).

44. Tavakkoli, K. G. et al. Sacrificial-post templating method for block copolymer self-Aasembly. Small 10, 493-499 (2014).

45. Sivaniah, E., Hayashi, Y., Iino, M., Hashimoto, T. \& Fukunaga, K. Observation of perpendicular orientation in symmetric diblock copolymer thin films on rough substrates. Macromolecules 36, 5894-5896 (2003).

46. Yager, K. G. et al. Disordered nanoparticle interfaces for directed self-assembly. Soft Matter 5, 622-628 (2009).

47. Park, S. et al. Macroscopic 10-terabit-per-square-inch arrays from block copolymers with lateral order. Science 323, 1030-1033 (2009).

48. Hong, S. W. et al. Unidirectionally aligned line patterns driven by entropic effects on faceted surfaces. Proc. Natl Acad. Sci 109, 1402-1406 (2012).

49. Peng, M., Ma, S., Hu, J. \& Wang, R. Hierarchical nanostructures of diblock copolymer thin films directed by a saw-toothed substrate. Soft Matter 11, 6642-6651 (2015).

50. Fasolka, M. J., Banerjee, P., Mayes, A. M., Pickett, G. \& Balazs, A. C. Morphology of ultrathin supported diblock copolymer films: theory and experiment. Macromolecules 33, 5702-5712 (2000).

51. Knoll, A. et al. Phase behavior in thin films of cylinder-forming block copolymers. Phys. Rev. Lett. 89, 035501 (2002). 
52. Stein, G. E., Kramer, E. J., Li, X. \& Wang, J. Layering transitions in thin films of spherical-domain block copolymers. Macromolecules 40, 2453-2460 (2007).

53. Ramírez-Hernández, A., Liu, G., Nealey, P. F. \& de Pablo, J. J. Symmetric diblock copolymers confined by two nanopatterned surfaces. Macromolecules 45, 2588-2596 (2012).

54. Jin, Z. \& Fan, H. Self-assembly of nanostructured block copolymer nanoparticles. Soft Matter 10, $9212-9219$ (2014).

55. Cao, X., Zhang, L., Gu, J., Wang, L. \& Lin, J. Designing three-dimensional ordered structures from directed self-assembly of block copolymer films in topographical templates. Polymer 72, 10-20 (2015).

56. Yan, N., Zhu, Y. \& Jiang, W. Self-assembly of ABC triblock copolymers under 3D soft confinement: a Monte Carlo study. Soft Matter 12, 965-972 (2016).

57. Takahashi, H. et al. Defectivity in laterally confined lamella-forming diblock copolymers: thermodynamic and kinetic aspects. Macromolecules 45, 6253-6265 (2012).

58. Kim, B. et al. Thermodynamic and kinetic aspects of defectivity in directed selfassembly of cylinder-forming diblock copolymers in laterally confining thin channels. J. Appl. Polym. Sci. 131, 40790 (2014).

59. Li, W. \& Müller, M. Thermodynamics and kinetics of defect motion and annihilation in the self-assembly of lamellar diblock copolymers. Macromoleclules 49, 6126-6138 (2016).

60. Matsen, M. W. \& Thompson, R. B. Equilibrium behavior of symmetric ABA triblock copolymer melts. J. Chem. Phys. 111, 7139-7146 (1999).

61. Pochan, D. J. et al. Toroidal triblock copolymer assemblies. Science 306, 94-97 (2004).

62. Sing, C. E., Zwanikken, J. W. \& Olvera de la Cruz, M. Electrostatic control of block copolymer morphology. Nat. Mater. 13, 694-698 (2014).

63. Park, W. I. et al. Host-guest self-assembly in block copolymer blends. Sci. Rep. 3, 3190 (2013).

64. Yager, K. G., Lai, E. \& Black, C. T. Self-assembled phases of block copolymer blend thin films. ACS Nano 8, 10582-10588 (2014).

65. Daoulas, K. C. et al. Fabrication of complex three-dimensional nanostructures from self-assembling block copolymer materials on two-dimensional chemically patterned templates with mismatched symmetry. Phys. Rev. Lett. 96, 036104 (2006)

66. Stoykovich, M. P. et al. Directed assembly of block copolymer blends into nonregular device-oriented structures. Science 308, 1442 (2005).

67. Stein, A., Wright, G., Yager, K. G., Doerk, G. S. \& Black, C. T. Selective directed self-assembly of coexisting morphologies using block copolymer blends. Nat. Commun. 7, 12366 (2016).

68. Jung, H. et al. Three-dimensional multilayered nanostructures with controlled orientation of microdomains from cross-linkable block copolymers. ACS Nano 5, 6164-6173 (2011).

69. Woo, S., Wang, H. S., Choe, Y., Huh, J. \& Bang, J. Three-dimensional multilayered nanostructures from crosslinkable block copolymers. ACS Macro Lett. 5, 287-291 (2016).
70. Müller-Buschbaum, P. GISAXS and GISANS as metrology technique for understanding the 3D morphology of block copolymer thin films. Eur. Polym. J. 81, 470-493 (2016).

\section{Acknowledgements}

Research carried out at the Center for Functional Nanomaterials, and the National Synchrotron Light Source II, Brookhaven National Laboratory, which are supported by the U.S. Department of Energy, Office of Basic Energy Sciences, under Contract No. DE-SC0012704. We thank the Dow Chemical Company for providing the chemical brush material.

\section{Author contributions}

A.R. and C.T.B. designed the original experiments. A.R. prepared samples, developed the fabrication protocols, characterized the samples with SEM and explored system parameter spaces. P.W.M. and G.D. assisted with refining experimental protocols. K.G.Y. and P.W.M. performed X-ray scattering characterization, and developed image analysis methods. K.G.Y. developed the mechanistic model. C.T.B. and K.G.Y. supervised the work. All authors contributed to data analysis, interpretation and manuscript preparation

\section{Additional information}

Supplementary Information accompanies this paper at http://www.nature.com/ naturecommunications

Competing financial interests: The authors declare no competing financial interests.

Reprints and permission information is available online at http://npg.nature.com/ reprintsandpermissions/

How to cite this article: Rahman, A. et al. Non-native three-dimensional block copolymer morphologies. Nat. Commun. 7, 13988 doi: 10.1038/ncomms13988 (2016).

Publisher's note: Springer Nature remains neutral with regard to jurisdictional claims in published maps and institutional affiliations.

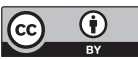

This work is licensed under a Creative Commons Attribution 4.0 International License. The images or other third party material in this article are included in the article's Creative Commons license, unless indicated otherwise in the credit line; if the material is not included under the Creative Commons license, users will need to obtain permission from the license holder to reproduce the material. To view a copy of this license, visit http://creativecommons.org/licenses/by/4.0/

C) The Author(s) 2016 